\title{
Extracción y evaluación de taninos condensados a partir de la corteza de once especies maderables de Costa Rica
}

\author{
Extraction and evaluation of condensed tannins from \\ bark of eleven species of trees from Costa Rica
}

\author{
Jorge Aguilar López' \\ Jean Carlo Jaén Jiménez² \\ Ana Sofía Vargas Abarca ${ }^{3}$ \\ Pablo Jiménez Bonilla ${ }^{4}$ \\ Ilena Vega Guzmán 5 \\ Jacqueline Herrera Núñez \\ Henry Borbón Alpizar? \\ Roy Mario Soto Fallas ${ }^{8}$
}

Fecha de recepción: 10 de febrero del 2012

Fecha de aprobación: 19 de abril del 2012

Aguilar, J; Jaén, J; Vargas, A; Jiménez, P; Vega, I; Herrera,

J, Borbón, H; Soto, R. Extracción y evaluación de

taninos condensados a partir de la corteza de

II especies maderables de Costa Rica Tecnología en Marcha. Vol. 25, № 4. Pág I5-22.

Químico. Universidad Nacional. Teléfono: 8323-6956

Correo electrónico: jorgealbertoaguilarlopez_89@hotmail.com

2 Químico. Universidad Nacional, Laboratorio de Manejo del Recurso Hídrico (LAMRHI). Teléfono: 2277-3824 Correo electrónico: jcarloj@hotmail.com

3 Química. Universidad Nacional, Laboratorio de Productos Naturales y Ensayos Biológicos (LAPRONEB). Teléfono: 8778-7388 Correo electrónico: sofiavarab@hotmail.com

4 Químico. Universidad Nacional, Laboratorio de Productos Naturales y Ensayos Biológicos (LAPRONEB). Teléfono: 89 | 6-2 I22 Correo electrónico: pabloijb@hotmail.com

5 Química. Universidad Nacional. Teléfono: 8729-5556 Correo electrónico: ilenave@gmail.com

6 Química. Universidad Autónoma de Madrid. Correo electrónico: herrerauna@gmail.com

7 Químico. Universidad Nacional. Teléfono: 8847-3529

Correo electrónico: hborbon.2000@gmail.com

8 Químico. Universidad Nacional. Teléfono: 8877-6843 Correo electrónico: roysoto@costarricense.cr 


\section{Palabras clave}

Taninos condensados, número de Stiasny, corteza, árboles de Costa Rica.

\section{Resumen}

La naturaleza química de los taninos condensados los convierte en una fuente natural de compuestos orgánicos, con una aplicación potencialmente amplia para usos medicinales e industriales.

La extracción y análisis de este tipo de metabolitos a partir de la flora de diversas regiones del planeta ha sido el objetivo del estudio de diversos grupos de trabajo, y en este caso se ha querido hacer un aporte al estado del conocimiento actual, realizando un estudio sobre la naturaleza y la cantidad del tanino condensado que se puede extraer de la corteza de II especies arbóreas presentes en Costa Rica: guanacaste (Enterolobium cyclocarpum), alcornoque (Licania arborea), jobo (Spondias mombin), pochote (Pachira quinata), níspero (Manilkara chicle), almendro (Andira inermis), roble (Tabebuia rosea), cedro (Cedrela odorata), cenízaro (Samanea saman), pino (Pinus caribaea) y ciprés (Cupressus lusitanica).

Las muestras de corteza fueron preparadas, secadas y extraídas con etanol. Se analizaron los extractos etanólicos para determinar el contenido de taninos condensados a través del número de Stiasny, y se caracterizaron mediante espectroscopía infrarroja (FT-IR).

Las especies con mayor proporción de material extraído fueron guanacaste $(9.5841 \% \mathrm{~m} / \mathrm{m})$, pochote $(15.0066 \%$ $\mathrm{m} / \mathrm{m})$, pino $(19.3400 \% \mathrm{~m} / \mathrm{m})$ y ciprés $(10.5300 \% \mathrm{~m} / \mathrm{m})$, mientras que los extractos con una mayor proporción de taninos condensados correspondieron a alcornoque $(61.9 \% \mathrm{~m} / \mathrm{m})$, jobo $(66.1 \% \mathrm{~m} / \mathrm{m})$, pochote $(72.8 \% \mathrm{~m} / \mathrm{m})$, níspero $(50.5 \% \mathrm{~m} / \mathrm{m})$, cedro $(72.7 \% \mathrm{~m} / \mathrm{m})$ y pino $(70.7 \%$ $\mathrm{m} / \mathrm{m})$.

\section{Key words}

Condensed tannins, Stiasny number, bark, costarrican trees.

\section{Abstract}

The chemical nature of condensed tannins offers a natural source of such organic compounds, with potential application to medicinal and industrial uses. The extraction and analysis of this type of metabolites from plants of many regions of the world have been the objective of researching groups.

In this case, it is desired to contribute to present knowledge, establishing the nature and amounts of condensed tannins extracted from bark of II tree species grown in Costa Rica: guanacaste (Enterolobium cyclocarpum), alcornoque (Licania arborea), jobo (Spondias mombin), pochote (Pachira quinata), níspero (Manilkara chicle), almendro (Andira inermis), roble (Tabebuia rosea), cedro (Cedrela odorata), cenízaro (Samanea saman), pino (Pinus caribaea) and ciprés (Cupressus lusitanica).

Bark samples of all mentioned species were prepared, dried and extracted with ethanol. Ethanolic extracts were analyzed to determine the condensed tannins content by Stiasny number and characterized by infrared spectroscopy (FT-IR).

The species with higher proportion of extracted material were guanacaste $(9.5841 \% \mathrm{w} / \mathrm{w})$, pochote $(15.0066 \% \mathrm{w} / \mathrm{w})$, pino $(19.3400 \% \mathrm{w} / \mathrm{w})$ and ciprés $(10.5300 \% \mathrm{w} / \mathrm{w})$, meanwhile extracts with higher proportions of condensed tannins were the obtained from alcornoque (61.9\%), jobo (66.1\%), pochote (72.8\%), níspero (50.5\%), cedro (72.7\%) and pino (70.7\%). 


\section{Introducción}

Los compuestos polifenólicos están presentes en los tejidos vegetales de muchas plantas (flores, frutos, semillas, hojas, raíces, madera y corteza), indistintamente de su ubicación geográfica (Rosales et al., 2002; Cano et al., 2002; Kim, 2009). La función de estos compuestos orgánicos dentro del metabolismo de las plantas no está claramente establecida. Sin embargo, su aprovechamiento abarca en la actualidad diversos usos, como la curtiembre de cuero, medicinales (antioxidantes), textiles (colorantes), formulación de adhesivos y síntesis de nuevos materiales, entre otros.

Dada su diversidad química y estructural, estos compuestos suelen clasificarse como taninos hidrolizables y taninos condensados. Los taninos hidrolizables son moléculas polifenólicas de tamaño discreto, de naturaleza no polimérica, capaces de ser hidrolizados por ácidos o enzimas a un azúcar y a un ácido carboxilico fenólico (Garro et al., 1997); mientras que los taninos condensados son moléculas oligoméricas o poliméricas de unidades flavan-3-ol, unidas a través de enlaces C-4 a C-8, o menos frecuentemente, C-4 a C-6 de dos unidades consecutivas (García et al., 1997), de diverso tamaño y constitución según su origen.

Una de las principales características de los taninos condensados es su capacidad de dar reacciones de condensación con formaldehído en medio ácido o básico (Kusak et al., 1998), dando como resultado un producto sólido polimérico con propiedades adhesivas (Taiwo, 1997; Wang et al., 1995), lo que constituye un método cuantitativo apropiado (Método de Stiasny) para la determinación gravimétrica de este tipo de metabolitos en cualquier tipo de extracto líquido o liofilizado (Suomi, 1985; Palma, 1990).

Actualmente, muchos investigadores centran sus estudios en la detección, análisis y cuantificación de metabolitos polifenólicos en diferentes tipos de especies vegetales, debido al auge que está experimentando la incorporación de sustancias de origen natural en la formulación de productos con aplicaciones clínicas e industriales (Soto et al., 200 I; Soto, 200 I). Es por ello que se ha suscitado un creciente interés en el estudio sistemático de compuestos polifenólicos presentes en la flora nacional, principalmente en la corteza de ciertas especies maderables, con el fin de dar una utilidad a los residuos gene- rados durante las operaciones de faenado y aserrío de especies que actualmente no se aprovechan en su totalidad.

La determinación de aquellas especies que proporcionen un mejor extracto de taninos, en términos de cantidad y calidad, será fundamental para el abordaje posterior de estudios relacionados con su potencial aplicación como precursores para la formulación de adhesivos derivados de la madera (Barboza et al., 20 I0; Pizzi, 1993).

\section{Materiales y métodos}

\section{Recolección de las muestras}

Los especímenes de las especies estudiadas se seleccionaron aplicando un muestreo aleatorio.

Las muestras de corteza de las especies jobo (Spondias mombin), pochote (Pachira quinata), níspero (Manilkara chicle), roble (Tabebuia rosea), cedro (Cedrela odorata) y cenízaro (Samanea saman) se recolectaron en el sector de Los Ángeles de Tilarán

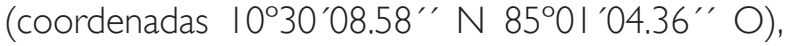
mientras que las muestras de las especies guanacaste (Enterolobium cyclocarpum), alcornoque (Licania arborea) y almendro (Andira inermis) se obtuvieron en el sector de Cañas (coordenadas 10²6 '24.63" N 8503'35.68" O) durante febrero del año 20। I; ambas localidades se ubican en la provincia de Guanacaste.

Por otra parte, las muestras de corteza de pino (Pinus caribaea) y ciprés (Cupressus lusitanica) se solicitaron durante el mismo periodo al aserradero de la Escuela de Ingeniería Forestal del Instituto Tecnológico de Costa Rica (ITCR), situado en la provincia de Cartago. Las muestras de corteza obtenidas de todas las especies estudiadas se extrajeron de la parte inferior de los respectivos troncos.

\section{Preparación y determinación del porcentaje de humedad de las muestras}

Las muestras de corteza recolectadas se limpiaron para eliminar restos de madera y se secaron durante siete días en un calentador de convección a 40 ${ }^{\circ} \mathrm{C}$. Las muestras secas se fragmentaron empleando un triturador de cuchillas marca Chicago, modelo 66910, y se molieron en un molino de cuchillas marca Retsch, modelo SMI00, utilizando una malla con un tamaño de abertura de $1 \mathrm{~mm}$. A todas las 
muestras se les determinó por triplicado el porcentaje de humedad, utilizando una balanza para humedad marca Adam, modelo PMB202.

\section{Extracción de taninos}

Realizando extracciones por triplicado, a $25 \mathrm{~g}$ de cada una de las muestras molidas se les adicionaron $200 \mathrm{~mL}$ de etanol al 95\%, dicha mezcla se puso a reflujo a $70{ }^{\circ} \mathrm{C}$ durante dos horas, con agitación continua. Al finalizar este periodo, las muestras se filtraron en caliente, al vacío, utilizando un papel Whatman \#54 para filtración rápida (tamaño de poro $22 \mu \mathrm{m})$.

Los extractos filtrados se concentraron en un evaporador rotatorio y se liofilizaron en un equipo Labconco Freezone 2.5, dentro de un matraz previamente tarado, para determinar por diferencia de masa la cantidad de material extraído de la muestra.

\section{Determinación del número de Stiasny}

Para la determinación del número de Stiasny, se pesó exactamente y por triplicado 0.I g de la muestra liofilizada en un erlenmeyer de $125 \mathrm{~mL}$ y se disolvió en $10 \mathrm{~mL}$ de metanol. A la mezcla resultante se le adicionaron I $\mathrm{mL}$ de ácido clorhídrico concentrado y $2 \mathrm{~mL}$ de formaldehído al 37\%, y se calentó a ebullición durante 30 minutos.

Transcurrido este tiempo, la mezcla fue filtrada al vacío en un embudo Büchner, empleando un papel filtro Whatman \#54, previamente tarado hasta masa constante, y una bomba de vacío marca KNF Laboport UN820.3FTP. El residuo sólido obtenido se secó a $105^{\circ} \mathrm{C}$ hasta alcanzar masa constante.

La masa de este sólido corresponde a la cantidad de tanino condensable presente en el extracto seco, y se expresa como un porcentaje respecto a la masa inicial del extracto liofilizado, de acuerdo con lo establecido en la expresión del número de Stiasny.

$$
\text { Número Stiasny }=\frac{\text { masa seca residuo sólido }}{\text { masa seca inicial muestra }} * 100
$$

\section{Análisis por espectroscopía infrarroja (FT-IR)}

Los extractos con un porcentaje de tanino superior al 70\% se caracterizaron por espectroscopía infrarroja, utilizando un espectrofotómetro infrarrojo con transformada de Fourier (FT-IR), marca Fisher Scientific, modelo Nicolet 1510, con el fin de confir- mar mediante esta técnica la naturaleza química del producto aislado, a partir de la identificación de los grupos funcionales presentes en las estructuras de los metabolitos obtenidos.

\section{Resultados y discusión}

Los datos de la determinación de los porcentajes de humedad para cada muestra se presentan en el cuadro I.

Los resultados obtenidos muestran que los contenidos de humedad de las muestras de corteza secas se encuentran alrededor de un valor del $10 \%$. Dicho valor coincide con el contenido de humedad presente en muestras de esta naturaleza en condiciones ambientales normales.

Además, se observa que aquellas cortezas que ofrecen un porcentaje de material extraible cercano o superior al 10\% corresponden a las especies de guanacaste, pochote, pino y ciprés, constituyéndose estas en las especies más promisorias del grupo estudiado para futuras evaluaciones de extracción y utilización de los taninos que de ellas se puedan extraer.

Por otra parte, los resultados obtenidos en la determinación del contenido de taninos condensados reactivos con formaldehído en medio ácido (número de Stiasny) sobre todos los extractos liofilizados se muestran en el cuadro 2.

Según Rosales et al. (2002), un material con un número de Stiasny superior a 65 es una buena fuente de taninos condensados para su posterior aplicación como adhesivos en productos celulósicos o de madera. Sin embargo, cuando se trabaja con matrices complejas como la corteza de los árboles, es necesario tomar en consideración no solo la cantidad de taninos condensados presentes en los extractos liofilizados sino también la cantidad de material extraído de la corteza, ya que esta es la fuente directa de estos metabolitos.

La combinación de ambos datos proporciona el valor real de la concentración de taninos presente en las muestras y permite seleccionar aquellas matrices que presentan una mayor cantidad de taninos condensados y por tanto mejores cualidades para ser utilizados como materiales de partida para la síntesis de adhesivos. 
Cuadro I. Porcentajes promedio de humedad y de material extraído de las muestras de corteza de las especies seleccionadas

\begin{tabular}{|c|c|c|}
\hline Especie & $\begin{array}{l}\text { Humedad promedio* } \\
\qquad( \pm 0.01 \% \mathrm{~m} / \mathrm{m})\end{array}$ & $\begin{array}{l}\text { Material extraído de la corteza* } \\
\qquad( \pm 0.0004 \% \mathrm{~m} / \mathrm{m})\end{array}$ \\
\hline $\begin{array}{c}\text { Guanacaste } \\
\text { (Enterolobium cyclocarpum) }\end{array}$ & 11.68 & 9.5841 \\
\hline $\begin{array}{c}\text { Alcornoque } \\
\text { (Licania arborea) }\end{array}$ & 9.77 & 4.6846 \\
\hline $\begin{array}{c}\text { Jobo } \\
\text { (Spondias mombin) }\end{array}$ & 6.62 & 3.1843 \\
\hline $\begin{array}{c}\text { Pochote } \\
\text { (Pachira quinata) }\end{array}$ & 12.63 & 15.0066 \\
\hline $\begin{array}{c}\text { Níspero } \\
\text { (Manilkara chicle) }\end{array}$ & 12.85 & 3.9629 \\
\hline $\begin{array}{c}\text { Almendro } \\
\text { (Andira inermis) }\end{array}$ & 11.18 & 5.5276 \\
\hline $\begin{array}{c}\text { Roble } \\
\text { (Tabebuia rosea) }\end{array}$ & 10.03 & $4.54 \mid 4$ \\
\hline $\begin{array}{c}\text { Cedro } \\
\text { (Cedrela odorata) }\end{array}$ & 12.85 & 5.0963 \\
\hline $\begin{array}{c}\text { Cenízaro } \\
\text { (Samanea saman) }\end{array}$ & 12.32 & 6.6891 \\
\hline $\begin{array}{c}\text { Pino } \\
\text { (Pinus caribaea) }\end{array}$ & 15.60 & 19.3400 \\
\hline $\begin{array}{c}\text { Ciprés } \\
\text { (Cupressus lusitanica) }\end{array}$ & 15.47 & 10.5300 \\
\hline
\end{tabular}

* Nivel de confianza del 95\%

Cuadro 2. Determinación del porcentaje de taninos condensados reactivos con formaldehído en medio ácido en los extractos liofilizados y la corteza de las especies estudiadas

\begin{tabular}{|c|c|c|}
\hline \multirow[b]{2}{*}{ Especie } & \multicolumn{2}{|c|}{ Porcentaje de taninos condensados reactivos } \\
\hline & $\begin{array}{l}\text { En el extracto liofilizado } \\
\qquad( \pm 0.1 \% \mathrm{~m} / \mathrm{m})\end{array}$ & $\begin{array}{l}\text { En la corteza } \\
( \pm 0.01 \% \mathrm{~m} / \mathrm{m})\end{array}$ \\
\hline $\begin{array}{c}\text { Guanacaste } \\
\text { (Enterolobium cyclocarpum) }\end{array}$ & 7.2 & 0.69 \\
\hline $\begin{array}{c}\text { Alcornoque } \\
\text { (Licania arborea) }\end{array}$ & 61.9 & 2.90 \\
\hline $\begin{array}{l}\text { Jobo } \\
\text { (Spondias mombin) }\end{array}$ & 66.1 & 2.10 \\
\hline $\begin{array}{c}\text { Pochote } \\
\text { (Pachira quinata) }\end{array}$ & 72.8 & 10.92 \\
\hline $\begin{array}{c}\text { Níspero } \\
\text { (Manilkara chicle) }\end{array}$ & 50.5 & 2.00 \\
\hline $\begin{array}{c}\text { Almendro } \\
\text { (Andira inermis) }\end{array}$ & 6.2 & 0.34 \\
\hline $\begin{array}{c}\text { Roble } \\
\text { (Tabebuia rosea) }\end{array}$ & 24.3 & 1.10 \\
\hline $\begin{array}{c}\text { Cedro } \\
\text { (Cedrela odorata) }\end{array}$ & 72.7 & 3.64 \\
\hline $\begin{array}{c}\text { Cenízaro } \\
\text { (Samanea saman) }\end{array}$ & 6.1 & 0.41 \\
\hline $\begin{array}{c}\text { Pino } \\
\text { (Pinus caribaea) }\end{array}$ & 70.7 & 13.67 \\
\hline $\begin{array}{c}\text { Ciprés } \\
\text { (Cupressus lusitanica) }\end{array}$ & 0.3 & 0.03 \\
\hline
\end{tabular}

* Nivel de confianza del 95\% 
Así, los resultados recopilados en el cuadro 2 muestran que las especies de pochote (Pachira quinata) y de pino (Pinus caribaea) ofrecen las concentraciones más altas de taninos condensados en corteza, correspondientes a $10.92 \% \mathrm{~m} / \mathrm{m}$ y $13.67 \% \mathrm{~m} / \mathrm{m}$ respectivamente. Dichos datos superan el valor límite de $8 \%$ establecido por algunos investigadores como parámetro para definir si la utilización de una madera o corteza es rentable para el aprovechamiento y extracción de los taninos condensados (Rosales et al., 2002).

Los espectros IR de los extractos secos obtenidos a partir de la corteza de pochote y de pino se muestran en las figuras I y 2.

El análisis de los espectros infrarrojo (FT-IR) de los extractos liofilizados derivados de la corteza de las especies Pachira quinata y Pinus caribaea muestran bandas intensas entre 3406 y $3224 \mathrm{~cm}^{-1}$, fuertes y anchas, características de los grupos hidroxilo $(-\mathrm{OH})$ presentes comúnmente en compuestos fenólicos. Otras señales a $1614 \mathrm{~cm}^{-1}$ y $1615 \mathrm{~cm}^{-1}$ son producto del alargamiento de los dobles enlaces carbono-carbono de las estructuras aromáticas presentes en este tipo de metabolitos (Pavia et al., 2009).

\section{Conclusiones}

Se evaluó el contenido de taninos condensados extraíbles de la corteza seca de II especies de árboles maderables de las regiones Norte y Central de Costa Rica, específicamente guanacaste (Enterolobium cyclocarpum), alcornoque (Licania arborea), jobo (Spondias mombin), pochote (Pachira quinata), níspero (Manilkara chicle), almendro (Andira inermis), roble (Tabebuia rosea), cedro (Cedrela odorata), cenízaro (Samanea saman), pino (Pinus caribaea) y ciprés (Cupressus lusitanica).

Las cortezas que presentaron mayor cantidad de material extraible fueron las de las especies de guanacaste, pochote, pino y ciprés. Sin embargo, en términos del contenido de taninos condensados en cada uno de los extractos obtenidos, las especies con mayores resultados fueron alcornoque, jobo, pochote, níspero, cedro y pino. Finalmente, las especies cuyas cortezas brindaron la mayor cantidad de extracto con los mayores contenidos de taninos condensados fueron el pochote y el pino.

Este resultado es muy importante, porque permitirá concentrar el interés de futuras investigaciones en el reconocimiento y evaluación de los taninos condensados presentes en estas especies, con el fin de encontrar posibles aplicaciones industriales, entre las

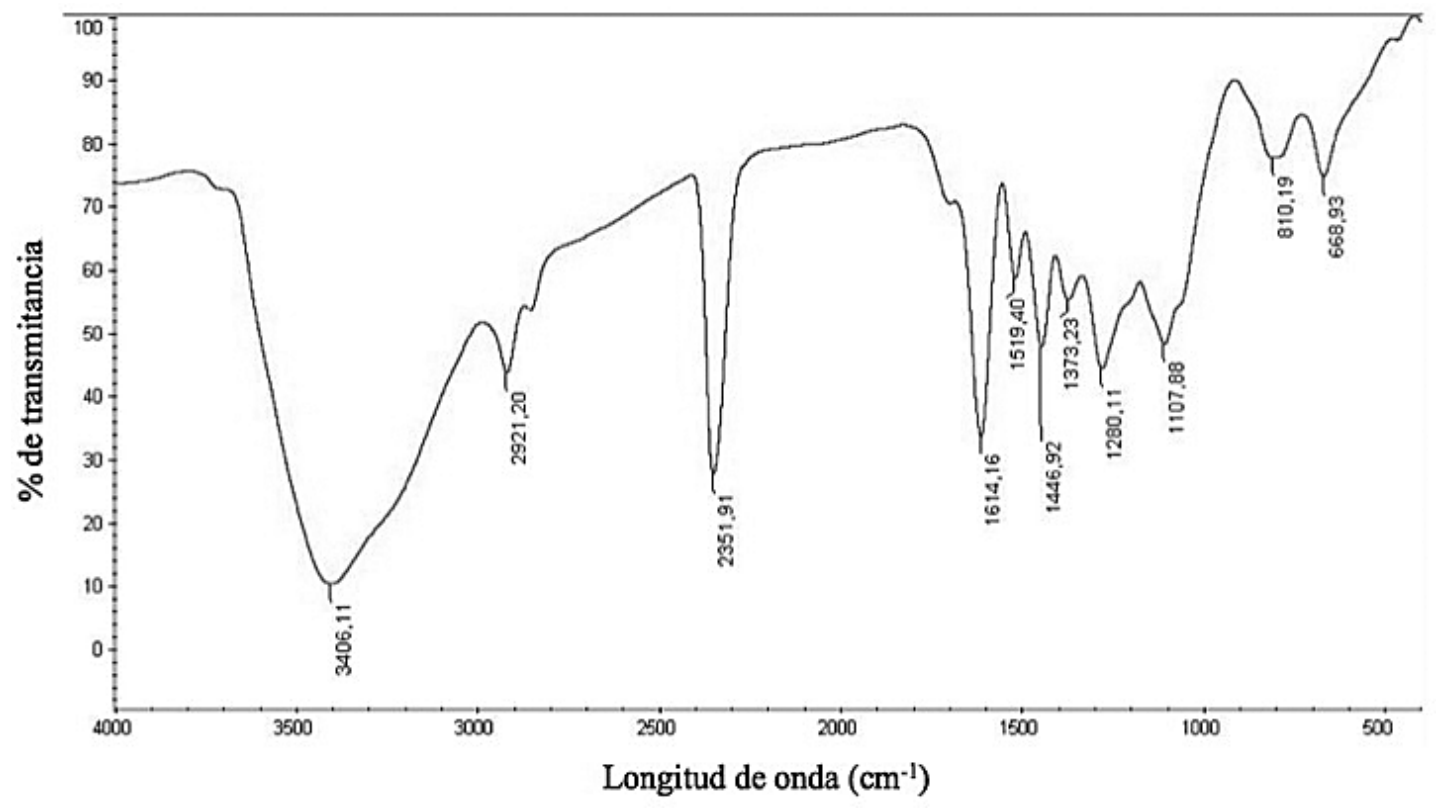

Figura I. Espectro de absorción infrarroja de extracto etanólico seco obtenido a partir de la corteza de pochote (Pachira quinata) 


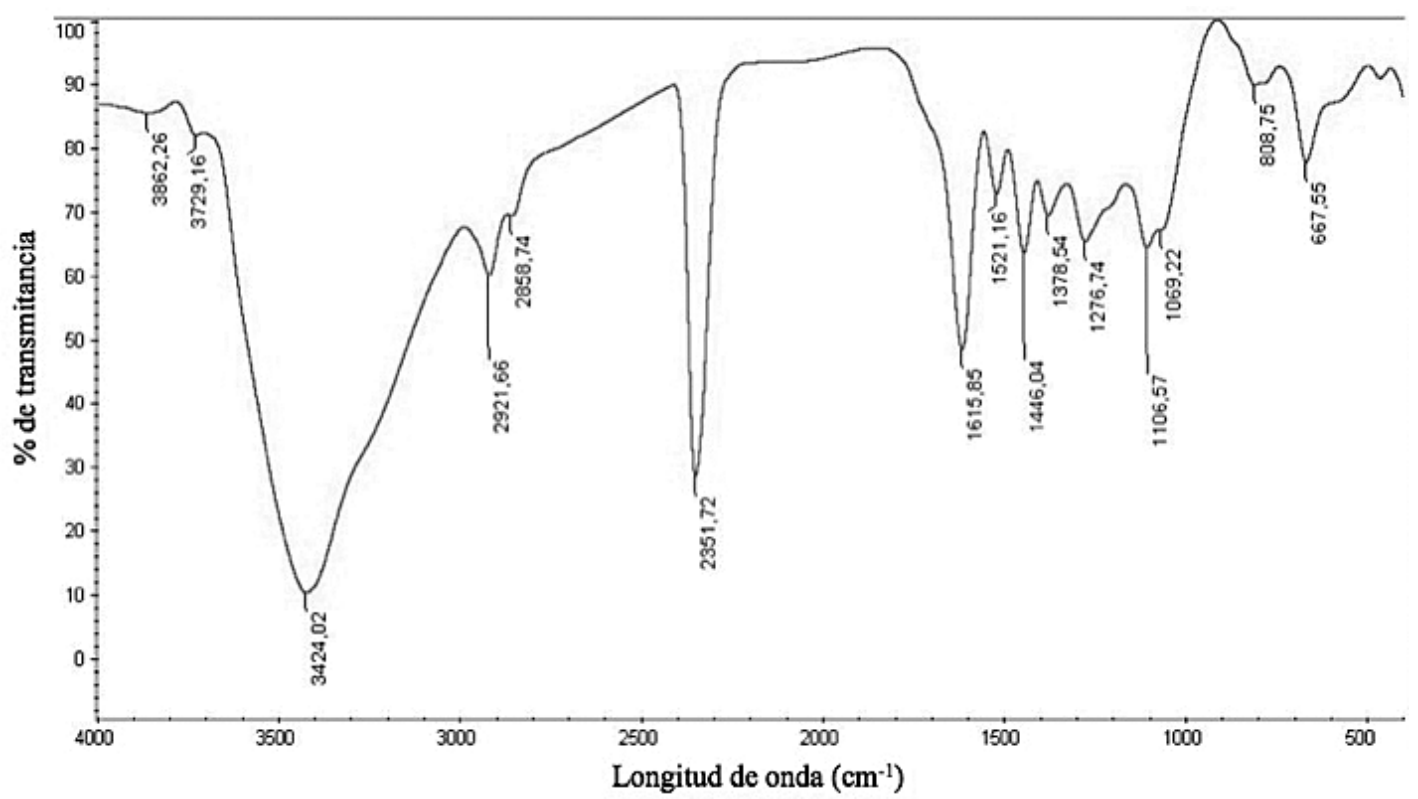

Figura 2. Espectro de absorción infrarroja de extracto etanólico seco obtenido a partir de la corteza de pino (Pinus caribaea)

cuales se vislumbra su aplicación en la formulación de adhesivos para madera, en sustitución de algunas sustancias químicas contaminantes que se utilizan en la actualidad para estos fines.

La naturaleza polifenólica de estos metabolitos queda confirmada a partir de los resultados observados por espectroscopía infrarroja (FT-IR).

\section{Agradecimientos}

Al Ing. Jorge Aguilar y a Isaías Jarquín, por su asistencia en el muestreo realizado en la Región Norte; al Dr. Róger Moya Roque y al Ing. Diego Camacho del Centro de Investigación en Integración Bosque Industria (CIIBI) de la Escuela de Ingeniería Forestal del Instituto Tecnológico de Costa Rica (TEC), por proporcionar las muestras de corteza de pino y ciprés utilizadas en este estudio.

\section{Bibliografía}

Barboza, V.; Frollini, E.; Ramírez, E. \& Tanaka, I. (20 I0). Biobased composites form tannin-phenolic polymers reinforced with coir fibers. Industrial Crops and Products 32: 305-312.
Cano, E.; Cano, T.; Quezada, O.; Quiñonez, B. \& Saravia, J. (2002). Extracción y caracterización de taninos en corteza de tres especies forestales cultivadas en Guatemala, Pino ocote (Pinus ooccarpa Schiede), Encino negro (Quercus brachystachys Benth) y Aliso común (Alnus jorulensis HBK). Una alternativa de desarrollo agroindustrial para el uso de taninos naturales. Guatemala: Carolina Academia Coactemalensis inter Caeteras Orbis Conspicua, pp. 5-6.

García, M.C.; Cadahía, E. \& Conde, E. (1997). Fifth Brazilian Symposium on the Chemistry of Lignins and Other Wood Components, Curitiba, Brasil.

Garro, J.M.; Riedl, B. \& Conner, A.H. (1997). Analytical studies on Tara tannins. Holzforschung 5 I: 235-243.

Kim, S. (2009). Environment-friendly adhesives for surface bonding of wood-based flooring using natural tannin to reduce formaldehyde and TVOC emission. Seoul: Department of Architecture, College of Engineering, Soongsil University (eds.), 745.

Kusak, S.; Hiltz, J. \& Waitkus, P. (1998). Impact performance of phenolic composities following thermal exposure. J. Appl. Polym. Sci. 67: 349-36I.

Palma, G.S. (1990). Caracterización y utilización de corteza de Pinus radiata D. Don (Tesis doctoral). 
Escuela de Graduados, Universidad de Concepción, Concepción, Chile.

Pavia, D.; Lampman, G. \& Kriz, G. (2009). Introduction to Spectroscopy. 4a. ed. Washington: Bellingham, Harcourt College Publishers.

Pizzi, A.; von Leyser, E.P.; Valenzuela, J. \& Clark, J.G. (1993). The chemistry and development of pine tannin adhesives for exterior particleboard. Holzforschung 47: I68- 174.

Rosales, M.; Galindo, A. \& González, R.F. (2002). Taninos condensados en la corteza de Pinus chihuahuana y Pinus durangensis. Información Tecnológica I3(1): 39-42.

Soto, R.; Freer, J.; Reyes, N. \& Baeza, J. (200I). Extraction of poliflavonoids from Pinus radiata $\mathrm{D}$. Don bark. Evaluation of effects of solvent compo- sition and of the height on tree bark. Bol. Soc. Chil. Quím. 46: 41 -49.

Soto, R. (200 I). Aislamiento, purificación, caracterización y modificación química de los taninos aislados de la corteza de Pinus radiata D. Don para su aplicación en la industria de las resinas y adhesivos para madera (Tesis doctoral). Escuela de Graduados, Universidad de Concepción, Concepción, Chile.

Suomi, L. (1985). Bark extracts and their use en plywood bonding. Pap. Puu 2: 65-69.

Taiwo, E. A. (1997). Bonding characteristics of adhesive/particle joint. Wood Sci. Technol. 31: 303-309.

Wang, X.M.; Riedl, B.; Christiansen, A.W. \& Geimer, R. L. (1995). The effects of Temperature and Humidity on phenol-formaldehyde resin bonding. Wood Sci. Technol. 29: 253-266. 